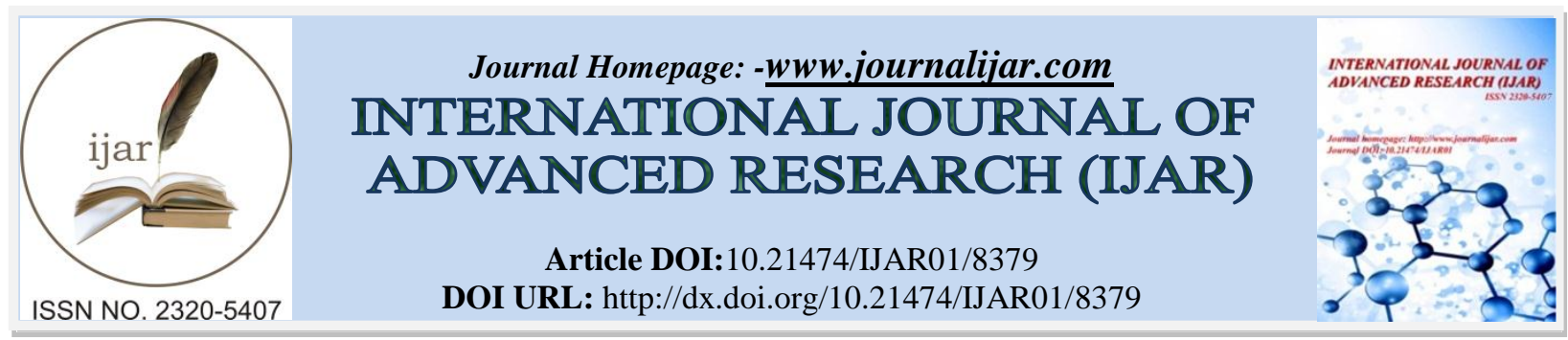

RESEARCH ARTICLE

\title{
LUDWIG'S ANGINA IN A 12 YEARS OLD GIRL.
}

Mazen Almasri.

King Abdulaziz University, Faculty of Dentisry, Oral Maxillofacial Surgery Department., Jeddah city, Saudi Arabia.

\section{Manuscript Info}

Manuscript History

Received: 10 November 2018

Final Accepted: 12 December 2018

Published: January 2019

Key words:-

Ludwig's angina, odontogenic, infection, space abscess, paediatrics.

\section{Abstract}

Ludwig's Angina (LA), is luckily a rare life-threatening medical situation faced by the medical personnel. It is rare in adult population and even less among paediatrics. A case report of LA that affected a 12 years old girl over a summer weekend is being reported and the literature review of similar cases in Saudi Arabia will be discussed.

Copy Right, IJAR, 2017,. All rights reserved.

\section{Introduction:-}

The infectious flare up of the submandibular, submental, and sublingual spaces leading to cellulitic attack and discharge space occupancy will have a tongue superior elevation and competing the upper airway entrance, and hence, it is a life threatening status. The former is the definition of Ludwig's Angina, that was first described back in 1836 (1). The infectious flare is usually accompanied by fever, luekocytosis, dehydration, dyspahagia, restlessness, and general malaise. Clinically the patients usually presented with significant indurated swelling of the neck, trismus, pain, and crusty dry lips that indicates malnutrition and dehydration (2). The status usually preceded by a milder form of the problem that is related to the origin of the infection. The origin can be odontogenic in nature or other non odontogenic sources such as tonsils, foreign body, glandular, epidermis, or others. (1)

The article is presenting a case of a 12 years old girl with LA presents to the Emergency department at King Abdulaziz Hospital, at the Southern part of Jeddah city, in Saudi Arabia.

\section{Case Report:-}

A 12 years old healthy Saudi girl presented at noon time on a weekend to the emergency department of King Abdulaziz Hospital at the Southern part of Jeddah city, in Saudi Arabia, crying and escorted by her mother, complaining of significant painful neck swelling, difficulty swallowing, and dysphonia (Fig. 1). There were not any signs of difficulty breathing at that point, and the vital signs were; blood pressure 100/65mmhg, heart rate was $90 \mathrm{bpm}$, and the temperature was 38.3 degree Cel. The oncall physician reported a history of tooth ache at the left mandibular molars during the last couple of weeks and the family was booked for dental appointment. She had had few doses of antibiotics that she is not aware of the name in addition to some analgesics.

As the clinical picture is presented and the urgency of the situation, laboratory tests were ordered immediately, CT scan, and starting intravenous fluids with empiric Antibiotic regimen.

The CT scan showed a 5x5cm left submandibular and sublingual large hypodense locules, with ring enhancement, and minor air pockets closer to the teeth, suggesting abscess collections. The cervical lymph nodes showed some enlargement and enhancements. The other muscles and fibrous envelops showed soft tissue swelling suggesting cellulitis changes (Fig 2,3). The source of the illness is likely to be the mandibular left first and second molars. The CBC came back proving leucocytosis of 19000 cell/micL, with elevated CRP and ESR.

Corresponding Author:-Mazen Almasri.

Address:-King Abdulaziz University, Faculty of Dentisry, Oral Maxillofacial Surgery 
The Diagnosis at that point was Ludwig's Angina due to odontogenic cause, and the plan was to manage the source immediately under general anesthesia, as considered to be an emergency intervention.

The OR team was consulted and the patient was cleared to be pushed to the OR holding area. After getting the consent forms signed and discussed, the patient was pushed to the OR room, and the anaesthetist prepared all the airway safety measures, and elected to intubate using the Glide scope which showed some abscess presence at the airway region and was suctioned immediately. Preoperative dexamethasone was administered and planned to be going for 48 hours.

The case then was prepared and draped in the routine manner for Oral Maxillofacial Surgical procedure. After the throat pack is placed, the mouth is opened wider to attempt dental extraction of the diseased teeth, mandibular left first and second molar were extracted and showed purulent discharge coming out of the sockets and through the floor of the mouth.

Next, the procedure was turned toward the neck swelling, two incisions were applied at a selected crease line below the mandibular inferior border of more than $2 \mathrm{~cm}$ to avoid the chance of injuring the marginal mandibular branch of the facial nerve. The first incision was placed at the submental region and the second was at the left submandibular region. Blunt dissections took place reaching the abscess two large locules that were safely entered and evacuated outward into a large kidney basin. Blunt dissection continued to reach the left ptyregomasseteric region, sublingual, vestibular, and communicating through the floor of mouth at the anterior and the left posterior aspects.

Significant saline irrigation was applied, squeezing, and massaging the neck to assure drainage and proper lavage. Multiple drains were inserted and secured intra and extraorally using Silk 3-0 sutures. A dressing was then applied and the patient was turned to the anaesthesia team, whom agreed to keep the patient intubated and referred to the ICU unit to continue the care.

The anaesthesia and ICU staff kept the patient intubated and sedated for the night to follow the airway, respiratory, and the systemic general status before deciding about extubation. The girl was extubated two days later after assuring the subside of the airway edema and the presence of an audible airway leak around the endotracheal tube (Leak test), with improved general situation, CT scan images, including the gradual withdrawal of the cervical drain until complete removal 48-72 hours postoperatively (Fig 4,5,6). She stayed in the ICU for four days and shifted to the ward to continue the care for another 5 days. She was on Ceftriaxone and Augmentin antibiotics throughout the period. The patient was discharged home after assuring stable recovery condition, stable vital signs, tolerating oral intakes, safe ambulation, voiding, and tolerable intra and extraoral wound discomfort.

Instructions of oral hygiene care, wound care, and nutrition was given, and recall visits continued for 6 weeks showing stable recovery.

Figures, Illustrations and captions:-

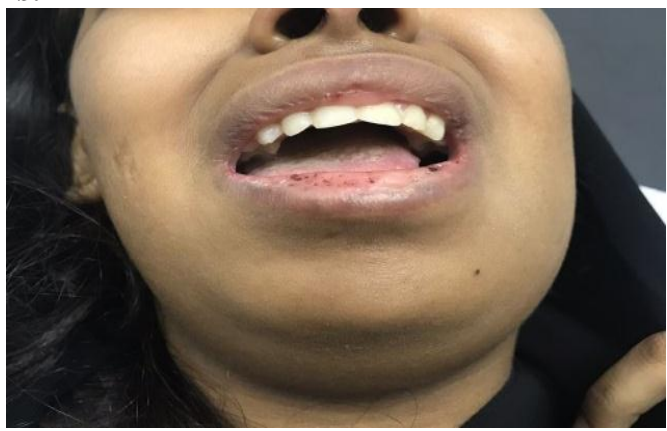

Figure 1:-a clinical picture showing the case as entered into the emergency room with swelling, trismus, and dry lips. 


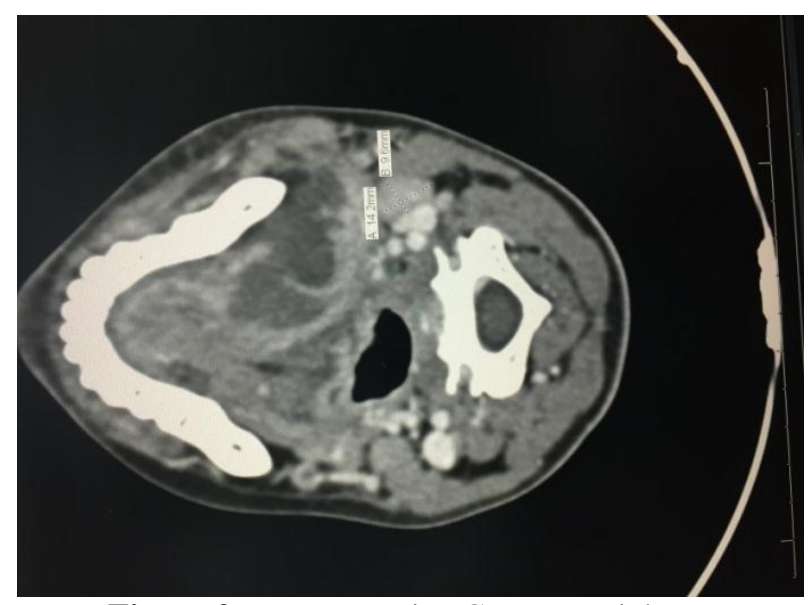

Figure 2:-a preoperative CT scan axial cut.

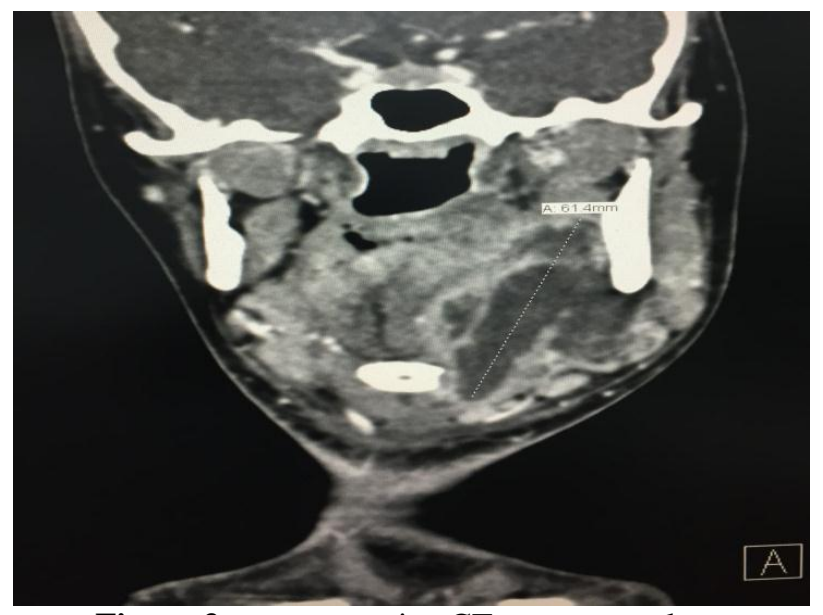

Figure 3:-a preoperative CT scan coronal cut

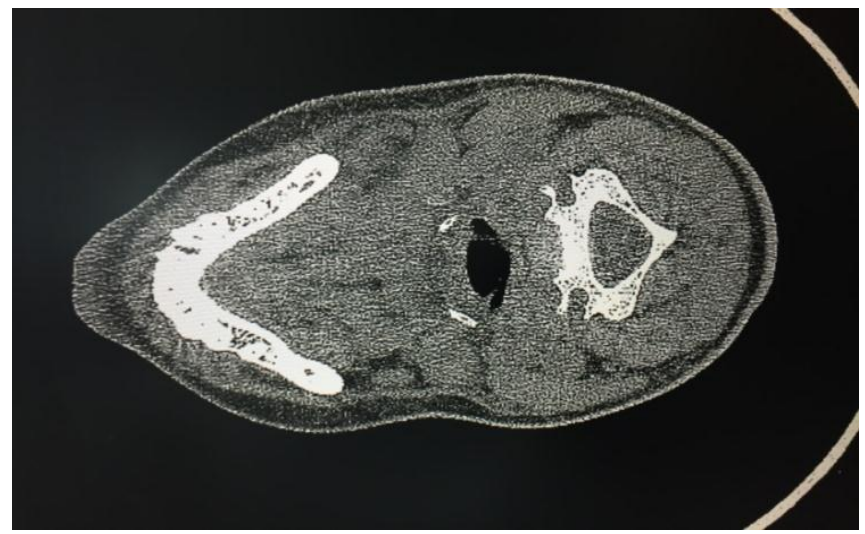

Figure 4:-a postoperative CT scan axial cut 


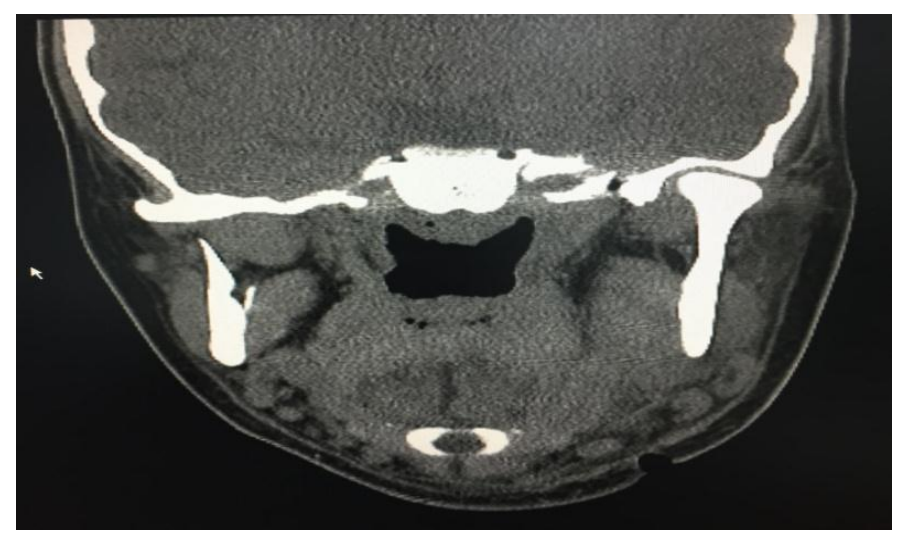

Figure5:-a postoperative CT scan coronal cut.

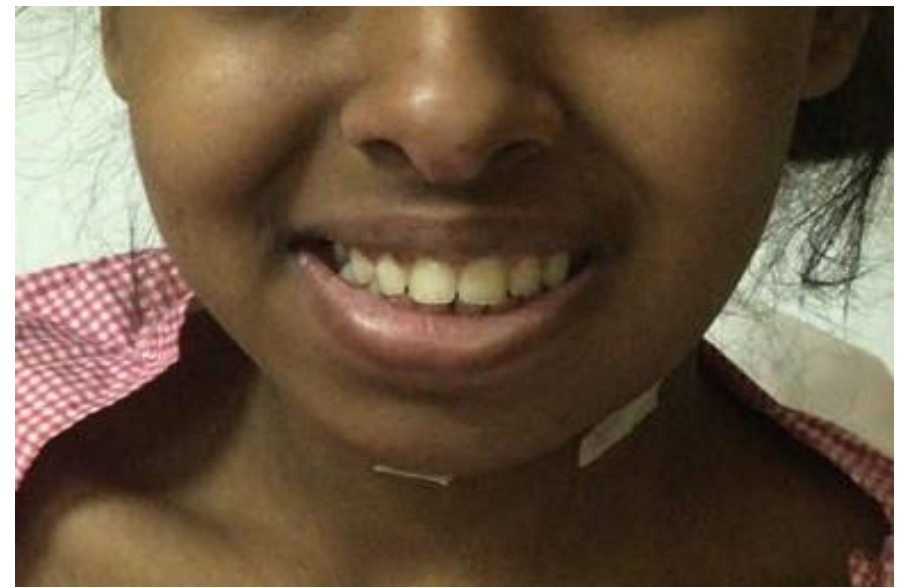

Figure 6:-a clinical picture showing the incision sites one week postoperatively and 4 days post drain removal.

\section{Discussion:-}

When searching through pubmed and Google scholar search engines using the keywords "pediatrics Ludwig's angina", they revealed numerous articles reaching 4800. When using more of the rule-in / out criteria to limit the search to the pediatric population, LA, cases in the same region, the search goes down to minimal number of articles with some relevance, to the best of my knowledge.

Neck Fascial space infections are cases that commonly seen in Oral Maxillofacial Surgery services. If the case of neck fascial space infection was neglected or did not receive the proper management, it might progress to include other neighboring spaces until it reaches all the neck spaces to be called, Ludwig's Angina (1). Luckily, cases are rarely postponed or neglected to reach this limit. The scenario of LA is even less common in children when compared to adults with no clear reported ratio in Saudi Arabia. Some studies suggested a ratio of 1:3.(2)

In addition to the dental or odontogenic source, LA can arise in children secondary to a variety of reasons such as tonsils, oral laceration, skin laceration, bug bites, jaw fracture, salivary gland disease, tumors, lymph nodes pathosis, foreign bodies, or secondary to herpetic gingivostomatitis. $(3,4,5)$

The situation can be potentiated as well with cases of weak immune status such Diabetis Meletus, sickle cell anemia, malnutrition, dehydration, or general sickness. (3)

In pediatric cases, the pathogen that is likely to be associated with LA are beta hemolytic streptococci and staphylococcus aureus $(3,6)$. The type of microorganism could not be specifically determined as the patient had started a random antibiotic regime with no clear information provided. 
Patients usually presented with their family after the acute flare of the case, that shows neck indurated painful swelling (Bull Neck), restlessness, malaise, trismus, dysphagia, dysphonia (hot potato voice), odynophagia, tongue swelling, difficulty of tongue movement, and difficulty of patient sleeping or even lying down as an active sign of airway space edema $(1,2)$. Younger children usually showed up carried by their parents, setting, or refuse to lye down with continuous restless crying or moaning $(3,4,5)$.

The cases of LA are considered as an emergency category one, and will require urgent decision-making process by experienced Oral Maxillofacial Surgeons, emergency physicians, and Anesthesiologists (7). And hence the importance of teaching the residents and the surrounding departments about that and the common signs and symptoms that might accompany the patients starting from a tooth-ache, neck swelling, pain, and odynophagia respectively (8). The case that was presented in the article is an example of immediately acting as multiple hospital team members to help out in managing the young girl. The cases do require precise recognition of the emergency and calling for the right tests. Furthermore, a fast and organized communication with the other team members such as anesthesia, ICU, ENT surgeons, and the OR team.

Due to the urgency of the airway, the chance of tracheostomy is always discussed during the consent form, however, it is rarely to be done for LA now days due to the improvement of endotracheal intubation techniques that included fiberoptic, glide scope, and awake intubation techniques. The experienced anesthetist is usually cautious in the way to approach such airway via general anesthesia to avoid any chances of rupturing the abscess or displacing the purulent discharge into the lower airway space leading to catastrophic outcomes including severe pneumonia, sepsis, or even death that recorded significant reduction now days down to $10-17 \%$, while it was reaching up to $50 \%$ in the past $(1,7)$.

Negligence or delayed management of pediatric minor dental infection might lead to fast progression into cellulitis and further spread into downward or upward directions (9). It can reach down to the mediastinal region or goes up toward the brain (10).

\section{Conclusion:-}

It is highly recommended to take dental infections seriously especially among the pediatric population. If the dental therapy did not help in managing the case and the swelling started to go beyond the vestibule, a consultation to Oral Maxillofacial Surgery department is advised. The flare up speed is totally unpredictable and hence the degree of management will differ significantly. Awareness campaign in schools and communities might help reaching out to parents to work more toward the prophylactic plan.

Acknowledgment:- The author would like to thank the administration of King Abdulaziz Hospital and Dr Ebtesam Aljerb ( Head of Oral Maxillofacial Surgery Department), for their ongoing support and care toward the emergency cases.

\section{Declaration:-}

The author declares no conflict of interests neither any funding for the article.

\section{References:-}

1. Jun-Kai Kao a, Shun-Cheng Yang. Ludwig's angina in children. Journal of Acute Medicine 1. 2011:23-26

2. Lin HW, Onerl A, Cunningham MJ. Ludwig's angina in the pediatric population. Clin pediatr (Phila). 2009;48(6):583-7.

3. Pandey M, Kaur M, Sanwal M, Jain A, Sinha SK. Ludwig's angina in children anesthesiologist's nightmare: Case series and review of literature. J Anaesthesiol Clin Pharmacol. 2017;33:406-9.E.A. Rosen, R.H. Schulman, A.S. Shaw. Ludwig's angina: a complication of a bilateral mandibular fracture: report of case. J Oral Surg. 1972;30:196-200

4. D.N. Lerner, T. Troost. Submandibular sialadenitis presenting as Ludwig's angina. Ear Nose Throat J. 1991;70:807-809

5. C.J. Chen, Y.C. Huang, T.Y. Lin. Ludwig's angina following herpetic gingivostomatitis in a toddler with tetralogy of Fallot. J Formos Med Assoc. 2004;103:311-313

6. Khalid Alnoori, Alsaid Lotfy. Deep neck spaces radiology and review of deep neck infections at King Abdulaziz University Hospital. EJENTAS. 2010;11:69-74 
7. Y. Chou, C. Lee, H. Chao, Hai-Hsuan Chao. An upper airway obstruction emergency. Ludwig angina. Pediatr Emerg Care. 2007;23:892-896

8. Gaurav Kataria, Aditi Saxen, Sanjeev Bhagat, Baldev Singh, Isha Goyal, Saurabh Vijayvergia, Parul Sachdeva. Prevalence of odontogenic deep neck space infections (DNSI): a retrospective analysis of 76 cases of DNSI. International Journal of Otorhinolaryngology and Head and Neck Surgery. 2015;1(1):11-16

9. Abdulla AlMushait, Zainab Darwish, Nermin Helal. Odontogenic facial cellulitis in Children. JKAU Med. Sci. 2012;19(1):103-117

10. Manasia, N Madisi, A Bassily, J Oropello, R Kohli. Ludwig's Angina complicated by fatal cervicofascial and mediastinal necrotizing fasciitis. ID Cases. 2016;4:32-33. 